\title{
Fuzzy Based Combinatorial Filter Model for Drusen Enhancement in Retinal Fundus Images
}

\author{
Jeyakarthikeyan, C, C Jayakumari
}

\begin{abstract}
This article presents an approach to enhance drusen regions in retinal fundus image of a patient having Age-related Macular Degeneration (AMD). In this approach, a new filter model is developed by combining two processes on images which is named as Combinatorial Filter Model (CFM). The first process is based on processing drusen significant image bit planes and the second process is based on implementing fuzzy inference system (FIS) on bit planes. When bit planes are independently processed, the result improves the visibility of drusen features. An FIS is constructed to process the bit planes to further enhance the processed image. This approach is tested on images with drusen features on good quality images in proprietary database and comparatively low quality images from STARE database. The objective study of this model shows that drusen elements are enhanced and validated to a $95 \%$ significant level. The quality of the enhanced image is evaluated for preservation of drusen features using a proven feature similarity index technique with 0.99 quality index values.
\end{abstract}

Keywords-Bit plane; filter; image processing; fuzzy inference system; image enhancement.

\section{INTRODUCTION}

Age related Macular Degeneration (AMD) is an eye disease that generally affects people in the age group beyond 55. The disease is caused because of one of a basic lesion called as druse or drusen. Macula is the central part of the retina having specialized layers of cells, Bruch's membrane, Retinal Pigment Epithelium (RPE) and choroid. The retina cells release waste by-products which are accumulated as drusen between RPE and Bruch's membrane. The two different types of drusen are hard drusen that is small with well-defined boundaries and soft drusen that is large with un-clear boundaries. ${ }^{1}$ The retinal image is captured as a fundus image using a special camera called fundus camera.

In India, the prevalence of AMD is constantly increasing over recent years, and is becoming very challenging for eye care professionals to treat those ailments. AMD in India ranges from $1.4 \%$ to $3.1 \%$ with $3.1 \%$, the highest in South India. The disease is predominant in people living in rural areas at about $2.3 \%$ than in urban areas at about $2.1 \%$. It is also found that the ailment affects more females $(2.5 \%)$ than males $(1.9 \%)$ in the age group above $65 .^{2}$

Revised Manuscript Received on July 10, 2019.

Jeyakarthikeyan, C, School of Advanced Career Education, SSN Institutions, Kalavakkam, Chennai - 603110, Tamil Nadu, India. (jkarthikn@gmail.com)

Dr C Jayakumari, Middle East College, Oman.

(jayakumaric@gmail.com)
In this study, we propose an innovative approach to create a model to enhance drusen features in retinal fundus images. The approach is trustable to a higher degree by validating on two databases.

\section{Data Set and Tool}

A total of 120 images were collected from two different medical image sources. (i) A private Eye Care Hospital donated 95 AMD images including 15 normal images of 768 by 546 pixel size. The ground truth images given by the hospital show manually marked different forms of drusen elements using their grading protocols. (ii) A count of 25 images is randomly picked from STARE database ${ }^{3}$ for validating our approach. The size of these images is of 700 by 605 pixels.

\section{Challenges \& Literature}

\subsection{Challenges}

There are various challenges involved in analysing fundus images for lesions. The challenges can be classified into two categories. In the first category, the type and size of druse lesions, in dry and wet AMD causes the difficulty in processing the image appropriately. The dry druse lesions are classified as early drusen, intermediate drusen and advanced drusen. ${ }^{4}$ This research focuses on any size of druse lesions with different pixel intensities. The next category of challenges is the quality of fundus image with respect to noise, intensity, color, and illumination to discriminate different ailments in retina. The image quality affects both enhancement and segmentation of drusen elements in fundus images. There are many research techniques focussed on medical image enhancement and segmentation, proposed by various authors.

\subsection{Literature}

The authors proposed techniques to locate optic nerve and macula in retinal fundus images. They describe methods to determine anatomic structures with anomalies such as drusen with macular position. ${ }^{5}$ Mei Zhou and Et. Al. propose an image enhancement method to improve contrast and luminosity in color retinal image. They implement gamma correction in HSV color space and obtain a luminance gain matrix to enhance RGB channels. Contrast is enhanced in luminosity channel by applying contrast-limited adaptive histogram equalization. They enhance the image preserving naturalness of the images. ${ }^{6}$ The authors proposed a machine learning algorithm to detect and quantify drusen automatically on retinal images. They conclude that machine learning is capable of detecting high risk- and low

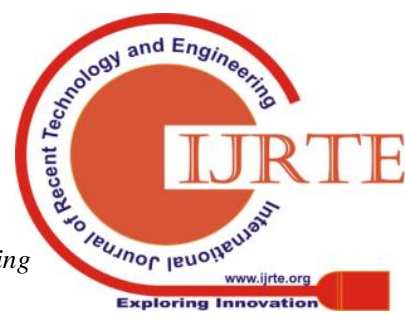


risk patients with non-advanced age related macular degeneration having drusen. Amplitude-modulation frequency-modulation decompositions for medical imaging problems including retinal image analysis is proposed by authors. In their research, they used a technique on AREDS images having retinal background, vessels, soft drusen and hard drusen elements for analysis. The standard deviations separating the histograms in retinal structures show that both soft and hard drusen are significantly differentiated with other retinal structures with the classification accuracy of more than $90 \%$. Moreover, the distance between soft and hard drusen types is found as 2.8 standard deviations. ${ }^{8}$

In research journal ${ }^{9}$, the authors propose a supervised featured learning to segment drusen from retinal images. The drusen features learnt through this method are vectored and trained with support vector machine to classify the pixels in test images as drusen or non-drusen. The training is based on drusen patches and background patches of AMD images. They evaluated the method on STARE and DRIVE databases with accuracy of $96.92 \%$ \& $94.81 \%$ respectively. The study is based on generalized low rank approximation and supervised term techniques. In article ${ }^{10}$, the authors De-focussing, averaging and max-min rule over the neighbours of a pixel were adopted to create overall improved enhancement of images. The effect of image quality is studied with linear index of fuzziness and entropy of the image. The authors proposed a grey prediction model to enhance image by modelling smoothness to data being sensitive to edges by implementing local contrast dynamically. ${ }^{11}$

Recent researches show that fuzzy inference system (FIS) is widely used in various research applications of digital image processing, specifically a significant role in retinal fundus image processing. One of the earlier researches is using fuzzy set theory on image enhancement, by fuzzy property plane pixel modification of an image. ${ }^{12}$

\section{PROPOSED APPROACH}

\subsection{Bit plane slicing}

Any greyscale image is composed of eight1-bit planes ranging from zero to seven. In retina images with AMD, drusen specific features are available in most significant bit plane and other relevant drusen information is also available in some of lesser significant bit planes which is discussed by authors in the article. ${ }^{13}$ This is explained in the following section. Each of these 1-bit planes will have planar information of an image. An image is sliced into 8 bit planes as shown in Fig. 1 and the right bottom corner image is the original color image.

\subsection{Drusen significance in bit planes}

Using trial and error method, for the given quality of fundus image, it is found that the $7^{\text {th }}, 5^{\text {th }}$ and $4^{\text {th }}$ planes have significant information pertaining to drusen element characteristics. In addition to useful information, insignificant information is also found in these planes. As a step to focus on drusen specific information, a combinatorial filter model is created composing functions of median and two dimensional digital filters. suggested greyscale image enhancement using fuzzy set.

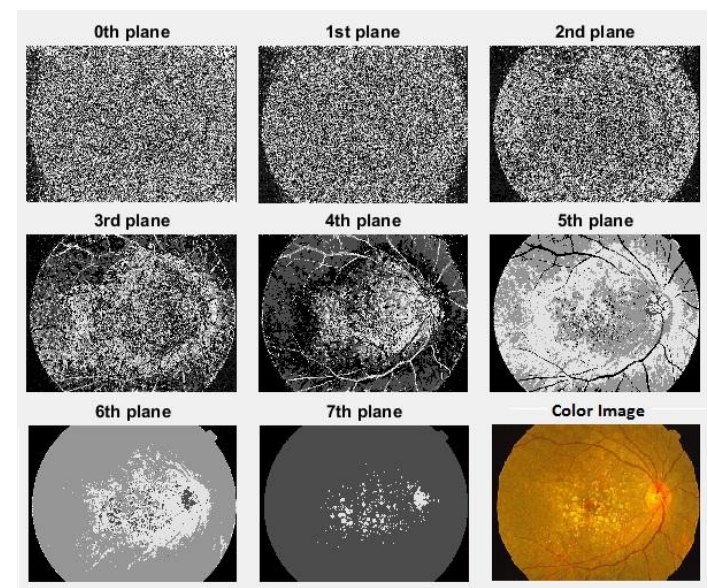

Fig. 1. Eight bit plane grey images and original color image

\section{COMBINATORIAL FILTER MODEL \& ANALYTICAL RESULTS}

\subsubsection{Pre-enhancement process}

The filter is designed in such a way that it minimizes the effect of filter operation on non drusen pixels. The median filter is applied to remove noises in initial images. The sliced plane image is given as an input to finite impulse response (FIR) filter with a filter matrix. The filter matrix is a threshold matrix, a structural element that is created specific to drusen elements in image. The FIR filter rotates filter matrix to 180 degrees in order to create a convolution kernel for the implementation of convolution function. This filter operation is applied to each of the selected bit planes and the output is generated as respectively modified new planes. It is found that the $7^{\text {th }}$ plane provides significant information specific to retinal drusen features. The $4^{\text {th }} \& 5^{\text {th }}$ planes are processed to improve the visibility of retinal background information in grey image plane. This is called as Pre-enhancement process.

The pre-enhancement process is done as described in the following 3 steps. In step 1, a filter function is applied with a $3 \times 3$ structuring element (SE1) to the $7^{\text {th }}$ plane of the image to create $\mathrm{f} 1$. Another $3 \times 3$ structuring element (SE2)is applied in the filter function to the same 7 th plane to create f2. Multiply f1 and $\mathrm{f} 2$ in order to improve visibility of drusen features. These functions are shown as expressions in Eq. (1). In step 2, a 3 x $\quad 3$ structuring element (SE3) is applied in the filter function to $5^{\text {th }}$ plane in order to process additional drusen feature in the image plane as shown in Eq. (2). In step 3, a 3 x 3 structuring element (SE4) is applied in the filter function to $4^{\text {th }}$ plane to improve the visibility of retinal background information as shown in Eq. (3) which is found to be subjective.

$$
\begin{aligned}
& \mathrm{P} 5=2 \operatorname{DFIR}(\mathrm{p} 5, \mathrm{SE} 3) . \\
& \mathrm{P} 4=2 \operatorname{DFIR}(\mathrm{p} 4, \mathrm{SE} 4) .
\end{aligned}
$$$$
\mathrm{P} 7=2 \mathrm{DFIR}(\mathrm{p} 7, \mathrm{SE} 1) \times 2 \mathrm{DFIR}(\mathrm{P} 7, \mathrm{SE} 2)
$$

\subsubsection{Plane processing}


The whole 8 bit image is reconstructed by composing the processed P7, P5\& P4 bit planes (ProcessedPlanes) in Eq. (4) and other planes p1, p2, p3, p6 as extracted from grey scale image. One of the disadvantage is that the impulse noise is introduced when these bit planes are processed. The fuzzy inference system (FIS) is created to remove these impulse noises and enhance the output image. This system is applied to three planes in combination to filter process. The enhanced image (EnhancedImage) is generated involving grey image (GreyImage) as shown in Eq. (4). The grey image is used to preserve other features of retina such as macula, nerves, optic disc including any other background information. The enhanced image shows retinal features with clarity that is subjectively evident.

EnhancedImage $=$ GreyImage +

$$
\text { (FIS(P7, P5, P4)) x ProcessedPlanes (P7, P5, P4). }
$$

(4)

\section{Fuzzy inference system}

In this model, these types of drusen are mapped to WeakDrusen, NormalDrusen and StrongDrusen.

\subsubsection{Role of fuzzy inference system}

The role of Fuzzy inference system is to identify the contribution of pixels to drusen regions in each of the three planes and process them accordingly. In FIS, three variables are used to fuzzy process each of the drusen significant bit plane images. The value bound for these input variables are set to 0.0 to 1.0 . Four members are added to each of the input planes implemented with Gaussian function in the fuzzification process.

\subsubsection{Membership Function}

FIS is a branch of artificial intelligence that uses logic and set theory concepts, known as fuzzy set theory. The fuzzy set theory uses membership functions to identify the degree of belongingness of a pixel to different kinds of drusen sets. Gaussian membership functions are applied for every drusen or non drusen types in each of the $4^{\text {th }}, 5^{\text {th }}$ and $7^{\text {th }}$ input bit planes. This is discussed in detail later in section 4.3.4.

\subsubsection{Fuzzy Rules}

A fuzzy rule is a rule that is used to infer an outcome based on input image. The canonical form of fuzzy rule is expressed as 'IF antecedent THEN consequent' in fuzzy rule based system. Mamdani fuzzy controller is a technique adopted in this research work. This controller uses fuzzy rules based on different drusen features which is designed to operate on each of the $4^{\text {th }}, 5^{\text {th }}$ and $7^{\text {th }}$ bit planes. These rules are applied to each of these bit planesduring fuzzification process. After fuzzification, the aggregated pixel values are created with 3 plane information. This is done in the defuzzification process. In this process, the output image variable is defined to de-fuzzify the fuzzy output to crisp output. That is, the bit plane pixel fuzzy intensity values are converted into crisp intensity values. The de-fuzzification process is implemented by applying triangular membership functions over a range of pixel intensity values for each drusen feature. Four fuzzy inference rules are generated and applied to these fuzzified planes to enhance drusen element pixels. A sample fuzzy rule is shown in Eq. (5).

If a pixel of bitPlane5 is NonDrusen then TestDrusen pixel feature is NonDrusen
The equation (5) is a rule, which states that if a pixel is processed as non drusen by FIS, then irrespective of same coordinate pixel values in other planes, the outcome pixel value is decided that it belongs to non drusen feature.

\subsubsection{Implementation of FIS}

The implementation of FIS is shown in Fig. 2. The drusen significant bit planes are given as crisp input to fuzzy process during fuzzification. This process creates fuzzy input which is processed by the inference engine. Fuzzy input and the fuzzy rules are used by the inference engine to infer image pixel values as fuzzy output values. In defuzzification process, the fuzzy output is de-fuzzified to generate crisp image pixel values using aggregation. In FIS, the crisp set theory describes that, $\mathrm{X}$ is a universal set of pixels in an image, $\mathrm{P}$ is the collection of well-defined pixels belonging to non drusen or drusen features (NonD, WD, $\mathrm{ND}, \mathrm{SD})$ and $x$ is a pixel element, where,

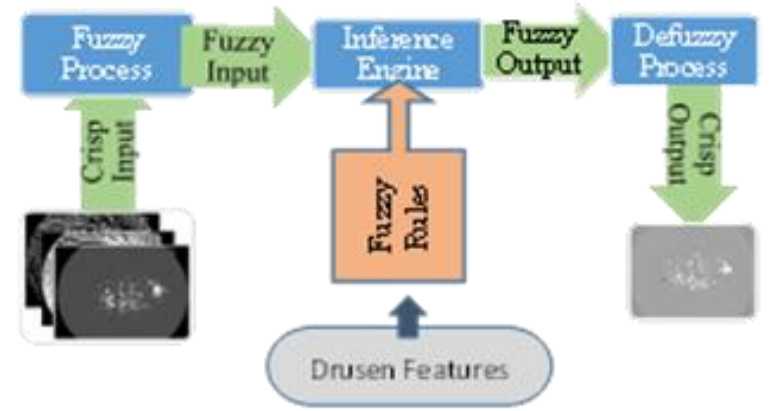

Fig. 2. Fuzzy Inference System

$x \in P$ or $x \notin P$.A Characteristic function of welldefined pixels in an image is expressed as

$$
\chi_{P}(x)=\left\{\begin{array}{l}
1, x \in P \\
0, x \notin P
\end{array}\right\} \text { where } \chi_{P}: X \rightarrow\{0,1\} \text {. The }
$$

theory is further described with the membership function expressed as $\mu_{P}: X \rightarrow\{0,1\}$. In fuzzy set theory, the set of all pixels in a well-defined set is expressed as $\left(x, \mu_{\bar{P}}(x)\right) \mid x \in X$. For example, the set of all pixels in well-defined ND is expressed as

$\left(x_{N D}, \mu_{\bar{P}}(x)\right) \mid x \in X$. Similarly, it can be defined for other sets NonD, WD \& SD. The membership function is expressed as

$\mu_{\bar{P}}: X \rightarrow[0,1]$. The grey pixel values are linearly

normalized in the range 0.0 to 1.0. These values are mapped to fuzzy set membership values in the range 0.0 to 1.0 . The alpha cut is expressed as $A_{\bar{\alpha}}=\left\{x \mid \mu_{\bar{P}} x \geq \alpha\right\}$ and $\alpha$ is arbitrary in $[0,1]$. The alpha cut values of normal drusen features (ND)

are identified as 0.43 to 0.76 corresponding to pixel values as shown in Fig. 4. These alpha cut values are found to be strong and is known as strong alpha cut values.

Four features are identified from graded images as, NonD refers to NonDrusen feature, WD refers to WeakDrusen feature, ND refers to 


\section{FUZZY BASED COMBINATORIAL FILTERMODEL FOR DRUSEN ENHANCEMENT IN RETINAL FUNDUS IMAGES}

NormalDrusen feature and SD refers to StrongDrusen feature. The membership function implementations are shown in Fig. 3. The (a) in Fig. 3 shows the pixel intensity values against degree of memberships for Gaussian input functions. From this Fig. 3, it is evident that the $7^{\text {th }}$ plane is having significant high order intensity information than $5^{\text {th }} \&$ $4^{\text {th }}$ planes. Next level high order intensity information is found in $4^{\text {th }}$ plane and lastly in $5^{\text {th }}$ plane.

The Fig. 4 shows the outcome of application of triangular membership functions to different intensity values in $7^{\text {th }}, 5^{\text {th }}$ and $4^{\text {th }}$ bit plane image as NonD, WD, ND and SD. The Figure also shows the amount of information available in each of the planes. Fig. 4 depicts that there are intersection points between NonD \& WD, WD \& ND, ND \& SD. These intersections show that there is increase in membership sharing of each of the feature from Non drusen to Strong drusen. It is shown that the smaller triangles formed by intersections on $\mathrm{x}$ axis are de-fuzzified as smoothened crisp values on subjective examination of images.
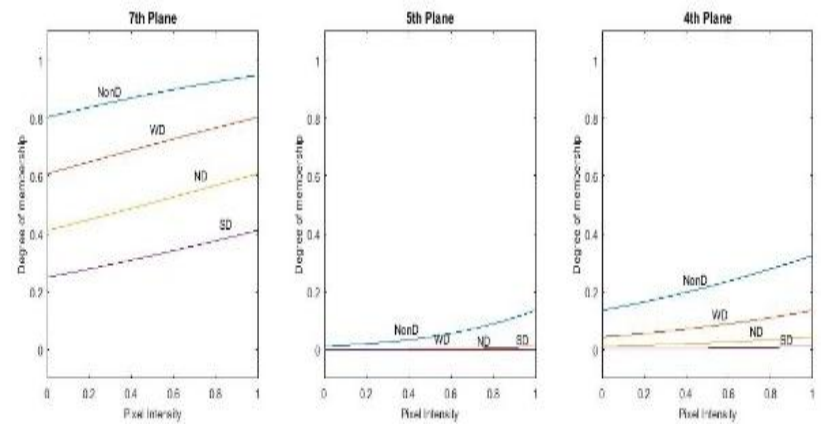

Fig. 3. (a) (b) (c) Pixel intensity and degree of pixel membership values in different bit planes in fuzzy process

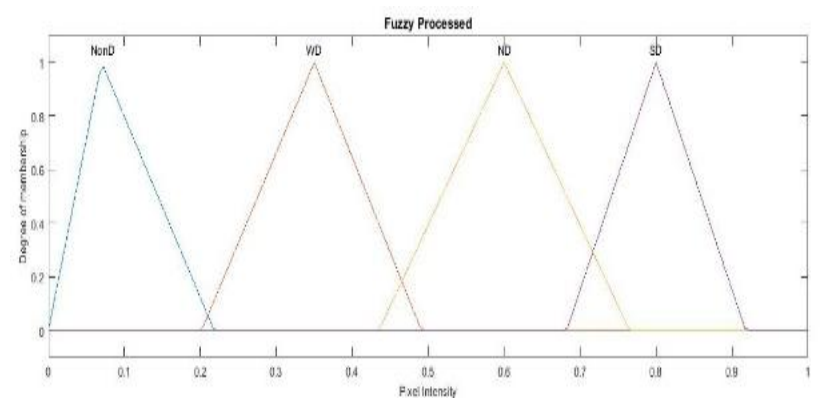

Fig. 4. Pixel intensity and degree of pixel membership of different drusen features inferred in fuzzy process

\subsection{Combinatorial Filter Model}

\subsubsection{CFM block diagram}

The block diagram in Fig. 5depicts the schematic process flowof the filtermodel. The input colorimage is converted to grey image and drusen significant planes are sliced. These slices are processed in two different modes, one with two dimensional median filter and the other with fuzzy inference system. The results are combined to generate an enhanced image.This model is known as combinatorial filter model.

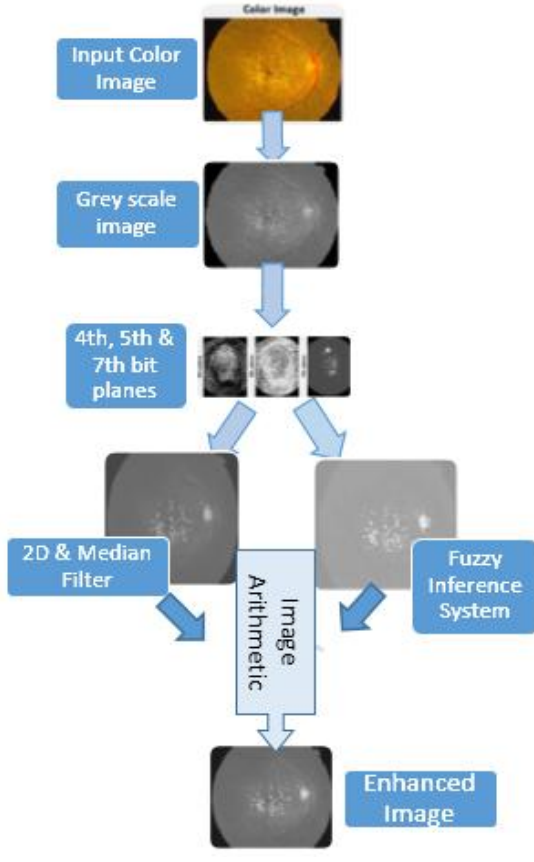

Fig. 5. Process flow diagram of Combinatorial Filter Model

\subsubsection{CFM algorithm}

1. Apply histogram matching with the RGB planes of the reference image to pre-process and convert it into grey scale image

2. Extract $4^{\text {th }}, 5^{\text {th }} \& 7^{\text {th }}$ bit planes of the grey scale image to start pre enhancement process

(a) Pre-enhancement step 1: Apply 2D digital filters pertaining to pre-enhance drusen features in the $7^{\text {th }}$ plane and create a new $7^{\text {th }}$ plane

(b) Pre-enhancement step 2: Apply two different and distinct 2D digital filters to 4th \&5th plane correspondingly to process drusen feature attributes

3. Re-construct the whole image by combining the independently processed $7^{\text {th }}, 5^{\text {th }}$ and $4^{\text {th }}$ planes with unprocessed other planes to get a partially enhanced drusen image

4. Construct a fuzzy inference system with the three planes $\mathrm{c} 4, \mathrm{c5} \& \mathrm{c} 7$ as input variables and one output variable with variable bounds set as zero and one

5. Fuzzify the stages of drusen features with the input variables to inference engine

6. Apply fuzzy rules to the inference engine to create fuzzy output image.

7. The fuzzified output images are de-fuzzified to generate a crisp output image

8. Use image arithmetic to fuzzy processed image with plane processed image to smoothen image features and improve visibility of AMD drusen elements Combine this image with original grey image 


\subsection{Results and Discussion}

\subsection{Pixel region process of drusen and non drusen regions}

The authors located different portions of retina image and compared the effect of contrast enhancement of drusenelements using fuzzy inference system. The comparisons were done using grey image, significant bit plane operated image, and the fuzzy processed image. Table1 shows different pixel region process information. This table shows information such as pixel region number, pixel window, Element (drusen / non drusen regions) those are selected for processing. The remaining columns show number of rows, number of columns and number of pixels processed. Error shows the number of pixels processed as drusen / non drusen appropriately. The false acceptance shows the percent of pixels in that region failed to process normally. The authors in ${ }^{14}$ proposed a color image enhancement method based on luminosity and contrast, which signifies that contrast of an image plays a vital role in image

\begin{tabular}{|c|l|l|c|c|c|c|c|}
\hline Region & \multicolumn{1}{|c|}{ Pixel Window } & \multicolumn{1}{|c|}{ Element } & Rows & Columns & Pixels & Error & False acceptance \\
\hline $\operatorname{Pr} 1$ & $\{335,271,342,274\}$ & Drusen & 4 & 8 & 32 & 1 & 3.125 \\
\hline $\operatorname{Pr} 2$ & $\{361,272,367,275\}$ & NonDrusen & 4 & 7 & 28 & 0 & 0.000 \\
\hline $\operatorname{Pr} 3$ & $\{562,82,570,85\}$ & NonDrusen & 4 & 9 & 36 & 0 & 0.000 \\
\hline $\operatorname{Pr} 4$ & $\{391,350,399,354\}$ & Drusen & 4 & 9 & 36 & 2 & 5.556 \\
\hline
\end{tabular}

In pixel region $1(\operatorname{Pr} 1), 32$ pixels are considered for process examination, and it is found that 1 pixel out of 32 is processed withdeteriorating pixel values. This deterioration is called as false rejection. It is also found that in region 4, 2 out of 36 pixels are processed as false rejection pixels. The accuracy of pixel enhancement is found to have $95.6 \%$ for these two regions.A consolidated false rejection rate post processing of 6 regions in 6 different images is found to have $3.98 \%$.

The representational image in Fig. 6 (a) shows the Region $1\{335,271,342,274\}$ marked as drusen in processed whole image and Fig. 6 (b) shows a blown up portion of post process mark of Region 1. The Fig. 7 (a) shows a sample grey scale image portion before processing and Fig. 7 (b) shows the processed image of the same portion.

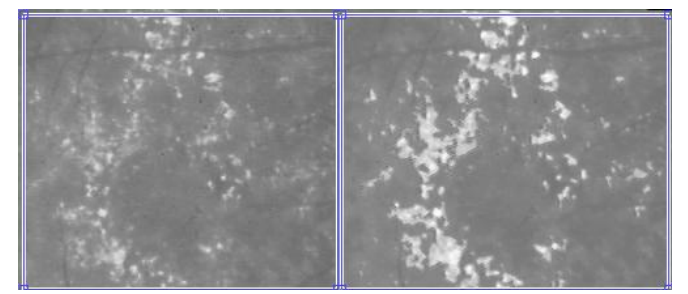

Fig. 7. (a) Drusen before applying CFM, (b) Image after applying CFM

Table 2. Row wise Intensity Deviation of Drusen Pixel Region 1

\begin{tabular}{|c|c|c|c|c|c|}
\hline Pixel Region & \multicolumn{2}{|c|}{ Mean Intensity } & \multicolumn{2}{|c|}{ Standard Deviation of Intensity } & \multirow{2}{*}{ Intensity Deviation } \\
\cline { 1 - 5 }$\{335,271,342,274\}$ & Input pixel & Processed Pixel & Input pixel & Processed Pixel & 0.086 \\
\hline Pr1 row1 & 0.564 & 0.650 & 0.047 & 0.115 & 0.080 \\
\hline Pr1 row2 & 0.571 & 0.651 & 0.043 & 0.101 & 0.084 \\
\hline Pr1 row3 & 0.576 & 0.660 & 0.060 & 0.112 & 0.084 \\
\hline Pr1 row4 & 0.576 & 0.660 & 0.065 & 0.102 & $\mathbf{0 . 0 8 3}$ \\
\hline Mean & $\mathbf{0 . 5 7 2}$ & $\mathbf{0 . 6 5 5}$ & $\mathbf{0 . 0 5 4}$ & $\mathbf{0 . 1 0 8}$ & $\mathbf{0 . 0 8}$ \\
\hline
\end{tabular}

Table 3. Row wise Intensity Deviation of Non Drusen Pixel Region 1

\begin{tabular}{|c|c|c|c|c|c|}
\hline Pixel Region 2 & \multicolumn{2}{|c|}{ Mean of Intensity } & \multicolumn{2}{|c|}{ Standard Deviation of Intensity } & \multirow{2}{*}{$\begin{array}{l}\text { Intensity } \\
\text { Deviation }\end{array}$} \\
\hline$\{360,271,368,276\}$ & $\begin{array}{l}\text { Input } \\
\text { pixel }\end{array}$ & $\begin{array}{l}\text { Processed } \\
\text { Pixel }\end{array}$ & Input pixel & Processed Pixel & \\
\hline Pr1 row1 & 0.429 & 0.470 & 0.009 & 0.006 & 0.041 \\
\hline Pr1 row2 & 0.42 & 0.466 & 0.008 & 0.005 & 0.046 \\
\hline Pr1 row3 & 0.421 & 0.464 & 0.004 & 0.005 & 0.043 \\
\hline Pr1 row4 & 0.421 & 0.470 & 0.004 & 0.0 & 0.049 \\
\hline Mean & 0.423 & 0.468 & 0.006 & 0.004 & 0.045 \\
\hline
\end{tabular}

Table 2 shows that for a graded pixel region with 4 rows, there is an overall increase in average 0.083 as the mean of intensity deviation and an overall increase in average 0.108 in the standard deviation of processed pixel. This indicates that the drusen regions are processed with good acceptance index. With reference to table 2, it is proved that there is an increase in contrast level of pixels in regions. Table 3 
shows the intensity values in processing NonDrusen portion in pixel region 2 .

It shows that 0.045 is the mean intensity deviation of input pixel and processed pixel for this region. Moreover, the standard deviation is calculated after processing intensity values with less average intensity value as 0.004 . The overall intensity deviation is calculated as 0.045 for all non drusen pixels in the region which indicates that the contrast is slightly improved for Non Drusen regions.

\subsection{Evaluation of Filter Model}

\subsubsection{Image and region sampling}

The stratified random probability sampling method is adopted in the selection process of images and regions to test. This is applied by dividing sample population images into different groups based on clinical characteristics categorized by the proprietary hospital. These images are used as representative images for testing the model. To evaluate the results of the model, drusen region area from each of images with ground truth information are selected for testing. The image was vertically divided in to two halves and a region is picked either from first half or the second half depending on drusen existence.

\subsubsection{Statistical test on drusen region comparisons}

Two drusen ailment regions were taken from six randomly picked images from proprietary database to prove the efficiency of the model in processing drusen regions. The F-test was done with the vectorized pixel values taken from the images after processing, to study the consistency in intensity variances. This test was carried out showing probability values of normal drusen pixels falling in the range 0.43520 .60 .7648 as shown in the fuzzy inference system membership function 'ND' in Fig.4. The comparisons were done with one drusen region selected from one image with a drusen region picked from another image. The table 4 shows the results of ten comparisons of different drusen regions from different images. The region nomenclature is also shown in this table.

The F-Test two sample for variances is used to test if the test statistic has an F-distribution with a Null hypothesis. The hypothesis is set as 'The variance of processed drusen region in one image is same as the variance of processed drusen region pixel in another image'. The alternate hypothesis is defined as 'The variance of processed region in one image is not the same as the variance of a region pixel in another image'. The study shows that the filter works better for eight comparisons out of ten in the same manner when it is implemented for different region comparisons from different images.

In hypothesis testing, 10 comparisons are shown in the table 4 . The probability values show that eight out of ten comparisons are having values greater than 0.05 which is the significant $p$ value. Two values are 0.0065 and 4.23E08 which are less than 0.05 for two comparisons.
Table 4. Comparison of different drusen regions from different images.

\begin{tabular}{|c|c|c|c|c|}
\hline \# & $\begin{array}{l}\text { Region } \\
\text { Name } \\
\end{array}$ & Nomenclature & $\begin{array}{l}\text { Regions of } \\
\text { two images }\end{array}$ & $\begin{array}{l}\text { Probability } \\
\text { of variance }\end{array}$ \\
\hline 1 & $1 \mathrm{r} 1-2 \mathrm{r} 1$ & $\begin{array}{l}\text { Image } 1 \\
\text { region } 1 \text { with } \\
\text { Image } 2 \\
\text { region } 1 \\
\end{array}$ & & 0.15 \\
\hline 2 & $3 r 1-1 r 1$ & $\begin{array}{l}\text { Image } 3 \\
\text { region } 1 \text { with } \\
\text { Image } 1 \\
\text { region } 1\end{array}$ & & 0.11 \\
\hline 3 & $3 r 1-1 r 2$ & $\begin{array}{l}\text { Image } 3 \\
\text { region } 1 \text { with } \\
\text { Image } 1 \\
\text { region } 2 \\
\end{array}$ & & 0.43 \\
\hline 4 & $3 r 2-1 r 1$ & $\begin{array}{l}\text { Image } 3 \\
\text { region } 2 \text { with } \\
\text { Image } 1 \\
\text { region } 1\end{array}$ & & 0.21 \\
\hline 5 & $4 \mathrm{r} 1-5 \mathrm{r} 1$ & $\begin{array}{l}\text { Image } 4 \\
\text { region } 1 \text { with } \\
\text { Image } 5 \\
\text { region } 1\end{array}$ & & 0.10 \\
\hline 6 & $5 r 1-6 r 1$ & $\begin{array}{l}\text { Image } 5 \\
\text { region } 1 \text { with } \\
\text { Image } 6 \\
\text { region } 1\end{array}$ & & 0.51 \\
\hline 7 & 6r1-1r1 & $\begin{array}{l}\text { Image } 6 \\
\text { region } 1 \text { with } \\
\text { Image } 1 \\
\text { region } 1 \\
\end{array}$ & & 0.16 \\
\hline 8 & $6 r 2-1 r 2$ & $\begin{array}{l}\text { Image } 6 \\
\text { region } 2 \text { with } \\
\text { Image } 1 \\
\text { region } 2 \\
\end{array}$ & 4. & 0.09 \\
\hline 9 & $1 \mathrm{r} 1-2 \mathrm{r} 2$ & $\begin{array}{l}\text { Image } 1 \\
\text { region } 1 \text { with } \\
\text { Image } 2 \\
\text { region } 2\end{array}$ & & 0.0065 \\
\hline 10 & 5r1-6r1 & $\begin{array}{l}\text { Image } 5 \\
\text { region } 1 \text { with } \\
\text { Image } 6 \\
\text { region } 1\end{array}$ & & 4.23E-08 \\
\hline
\end{tabular}

Hence, it is interpreted that the filter processing in different regions are statistically significant, which means that Null hypothesis is accepted for $80 \%$ comparisons. That is, 'The variance of processed drusen region in one image is same as the variance of a processed drusen region in another image' is accepted. Therefore, when the filter model processes different regions in different images, it is found that the model works with $95 \%$ significance level.

\subsubsection{Image quality}

Image quality is evaluated subjectively based on how the human visual system understands image with the low level features. ${ }^{15}$ In the proposed research article,

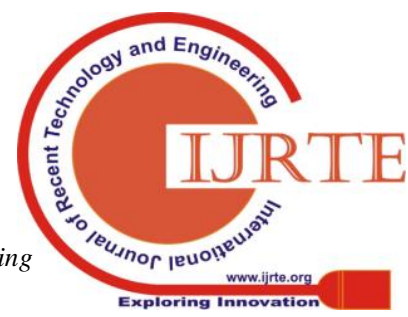


Fig. 7 (b) shows that the processed image region is subjectively known to have increased contrast than the pre-processed image in 7 (a). This is evaluated using visual perception.

The authors propose a phase congruency (PC) and image gradient magnitude (GM) features to characterize the image local quality. This method is called as Feature Similarity Index (FSIM) for full reference image quality assessment. In this paper, the luminance component of the image takes a vital role in image quality index. ${ }^{15}$ The Fig. 8 depicts quality test results showing FSIM index values for initial pre-process image compared with post fuzzy processed image for 60 images in proprietary database. The Fig. 9 shows the FSIM index values for 25 STARE images in subjective quality assessment.

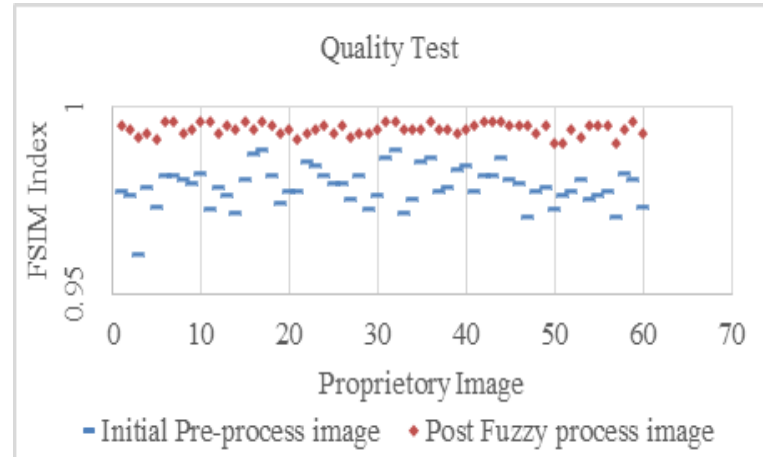

Fig. 8. FSIM index of pre-process and post process Images in Proprietary Image database

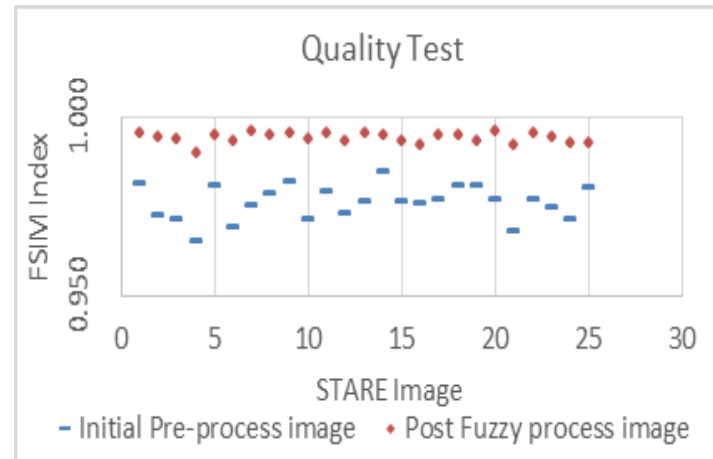

Fig. 9. FSIM index of pre-process and post process Images in STARE Image database

In these two figures, hyphen marked points refer to FSIM values of pre-processed images and diamond marked points refer to FSIM values of post processed images. It is evident that all post fuzzy processed images' FSIM values are greater than 0.990 tested in two databases. The average FSIM index for initial preprocessed images is 0.978 and that of post fuzzy processed images is 0.994 in proprietary database images. The average FSIM index for initial pre-processed images is 0.976 and that of post fuzzy processed images is 0.994 in Stare database images. The quality index values show that, the post processed images are preserved with good subjective quality features when compared to the respective pre-processed images.

\section{CONCLUSION}

In this research article, the authors proposed a novel drusen enhancement filter model. The model is a combination of bit plane process and fuzzy inference system processes called as combinatorial filter model. This model enhances the visibility of drusen portions in fundus images across two test databases one proprietary and another public STARE database. The enhancement is proved objectively with F-Test distribution. Post enhancement, the image quality is retained to a good extent. This ensures that other ophthalmic features such as optic disc, optic nerves, and fovea are also preserved to enable more efficient screening of drusen features in fundus images.

\section{ACKNOWLEDGEMENT}

The authors acknowledge the support given by SSN Institutions by providing research facility with Matlab R2018b software tool licence which enabled us to work on this research.

\section{REFERENCES}

1. Retina Institute of California, https://www.youtube.com/watch?v=74PIn4PYAoE

2. N. Likhar, R.K. Mothe, R. Kanukula, C. Shah, A. Dang, MarksMan, Healthcare Solutions LLP, 'The prevalence of age-related Macular degeneration in Indian Population: a systematic review', The Journal of The International Society for Pharmacoeconomics and Outcomes Research

3. STructured Analysis of the Retina (Stare), http://cecas.clemson.edu/ ahoover/stare/

4. National Eye Institute. Age-Related Eye Disease Study. http://www.nei.nih.gov/amd

5. Edward Chaum, V. PriyaGovindasamy, Thomas P. Karnowski, Kenneth W. Tobin, 'Detection of Anatomic Structures in Human Retinal Imagery', IEEE Transactions on Medical Imaging, 2007, vol. 26, issue 12, pp. $1729-$ 1739

6. Mei Zhou, Kai Jin, Shaoze Wang, Juan Ye \&DahongQian, 'Color Retinal Image Enhancement Based on Luminosity and Contrast Adjustment', IEEE Transactions on biomedical engineering, 2018, vol. 65, issue 3, pp. 521 - 527

7. Mark J. J. P. van Grinsven, Yara T. E. Lechanteur, Johannes P. H. van de Ven, Bram van Ginneken, Carel B. Hoyng, Thomas Theelen\& Clara I. S'anchez, 'Automatic Drusen Quantification and Risk Assessment of Age-Related Macular Degeneration on Color Fundus Images', Retina, April 2013

8. Victor Murray1, Marios S Pattichis, Eduardo Simon Barriga\& Peter Soliz, Murray et al., 'Recent multiscale AM-FM methods in emerging applications in medical imaging, EURASIP Journal on Advances in Signal Processing, 2012, 2012:23

9. XiuxiuRen, YuanjieZheng, Yanna Zhao, Chao Luo, Hong Wang, JianLian\&Yunlong He1, 'Drusen Segmentation From Retinal Images via Supervised Feature Learning', IEEE Access,December 2017, Digital Object Identifier 10.1109/ACCESS.2017.2786271

10. Sankar k. Pal and robert a. King, 'Image Enhancement Using Smoothing with Fuzzy Sets', IEEE Transactions on Systems, Man and Cybernetics, 1981, vol. SMC-1 1, no. 7

11. Gang Li, Image Contrast Enhancement Algorithm Based on $\operatorname{GM}(1,1)$ and Power Exponential Dynamic Decision, International Journal of Pattern 
Recognition and Artificial Intelligence, 2018, Vol. 32, No. 2

12. Sankar K. Pal and Robert A. King, Image Enhancement Using Smoothing with Fuzzy Sets, IEEE Transactions on Systems, Man, and Cybernetics, July 1981, vol. smc-1 1, no. 7

13. C. Jeyakarthikeyan, C. Jayakumari, A Bit Plane based Filtering Technique for Drusen in Age-related Macular Degeneration, Indian Journal of Science and Technology, 2016, Vol 9(48)

14. Mei Zhou, Kai Jin, Shaoze Wang, Juan Ye, DahongQian, Color Retinal Image Enhancement Based on Luminosity and Contrast Adjustment, IEEE Transactions on Biomedical Engineering, March 2018, Volume: 65, Issue: 3

15. Lin Zhang; Lei Zhang; XuanqinMou; David Zhang, A Feature Similarity Index for Image Quality Assessment, IEEE Transactions on Image Processing, August 2011, vol. 20, no. 8 